\title{
Universiteit
}

Leiden

The Netherlands

\section{Technology meets quantum criticality}

Zaanen, J.

\section{Citation}

Zaanen, J. (2005). Technology meets quantum criticality. Retrieved from https://hdl.handle.net/1887/5128

Version: $\quad$ Not Applicable (or Unknown)

License: $\quad$ Leiden University Non-exclusive license

Downloaded from: https://hdl.handle.net/1887/5128

Note: To cite this publication please use the final published version (if applicable). 


\section{SUPERCONDUCTIVITY}

\section{Technology meets quantum criticality}

Superconductivity and antiferromagnetism are in fierce competition in hightemperature superconductors. However, this competition has the unexpected benefit that the antiferromagnetism improves the capacity of the superconductor to resist magnetic fields.

\section{JAN ZAANEN \\ is at the Instituut-Lorentz for Theoretical Physics of Leiden University, 2300 RA Leiden, The Netherlands.}

e-mail: jan@lorentz.leidenuniv.nl

H igh-temperature superconductors made of cuprate-perovskite ceramics raised high expectations for revolutionary applications nearly 20 years ago (see Fig. 1 for a picture of the structure of these materials). But the initial frenzy turned into a hangover when it became clear that these materials were not useful for technical applications involving high magnetic fields, such as levitating trains. From a more fundamental viewpoint, it was quickly realized that the electron systems in these superconductors are of an entirely new kind. Their exotic behaviour arises from competition on a quantum-mechanical level involving collective electronic states such as antiferromagnetism, in which each copper spin is antiparallel to its nearest neighbour. However, on the technological front there was no apparent need to pay attention to these exotic matters that seemed to bear no apparent relevance to actual applications. But in this issue, Bella Lake et al. report the use of neutron scattering to study the antiferromagnetic fingerprints of this competition in applied external magnetic fields, to arrive at the conclusion that these exotic collective states do matter, and have the beneficial effect of immobilizing the magnetic fields penetrating the superconductor: the main goal of applied superconductivity ${ }^{1}$.

Superconductors are perfect conductors but, even more importantly, they expel magnetic fields. In 2003, the Russian theorist Abrikosov earned the Nobel Prize for his 1950s discovery that in certain superconductors magnetic fields enter in the form of quantized magnetic flux lines, inside which the electrons are not superconducting but normal ${ }^{2}$. Electromagnetic fields exert forces on these flux lines and their responding movement ruins perfect conduction. However, the flux lines form a solid-like lattice, a periodic array of magnetic flux lines that penetrates the superconducting material in the presence of a magnetic field. In order to move a part of this array one has to move the whole, and the whole easily gets stuck.

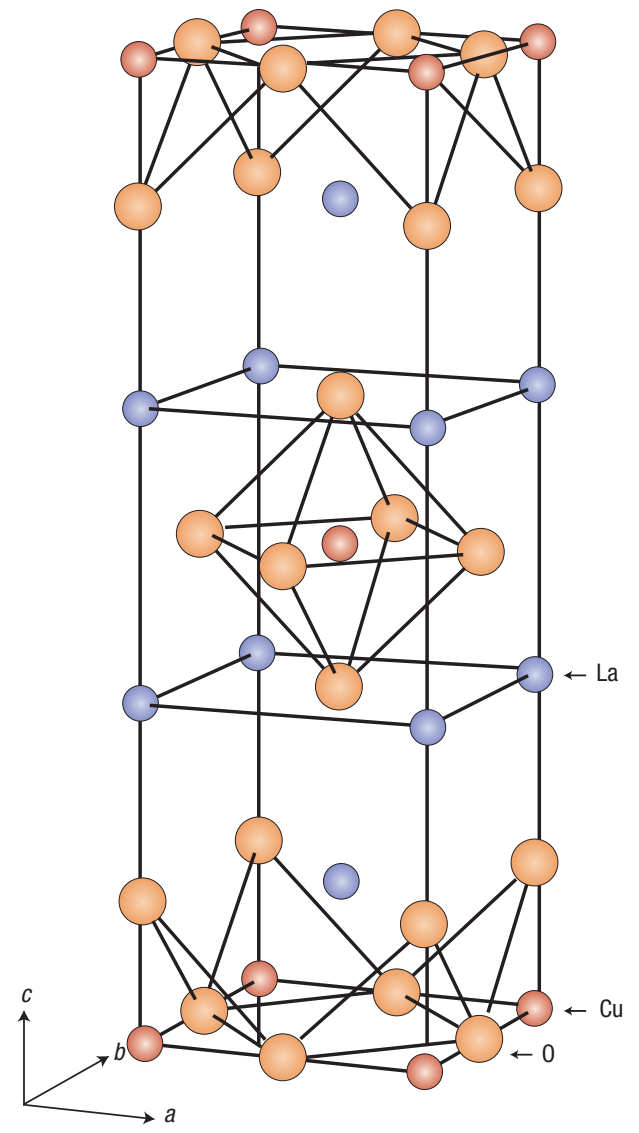

Figure 1 Crystal structure of $\mathrm{La}_{2} \mathrm{CuO}_{4}$. Superconductivity occurs in the $a b$-planes defined by the copper and oxygen atoms. 


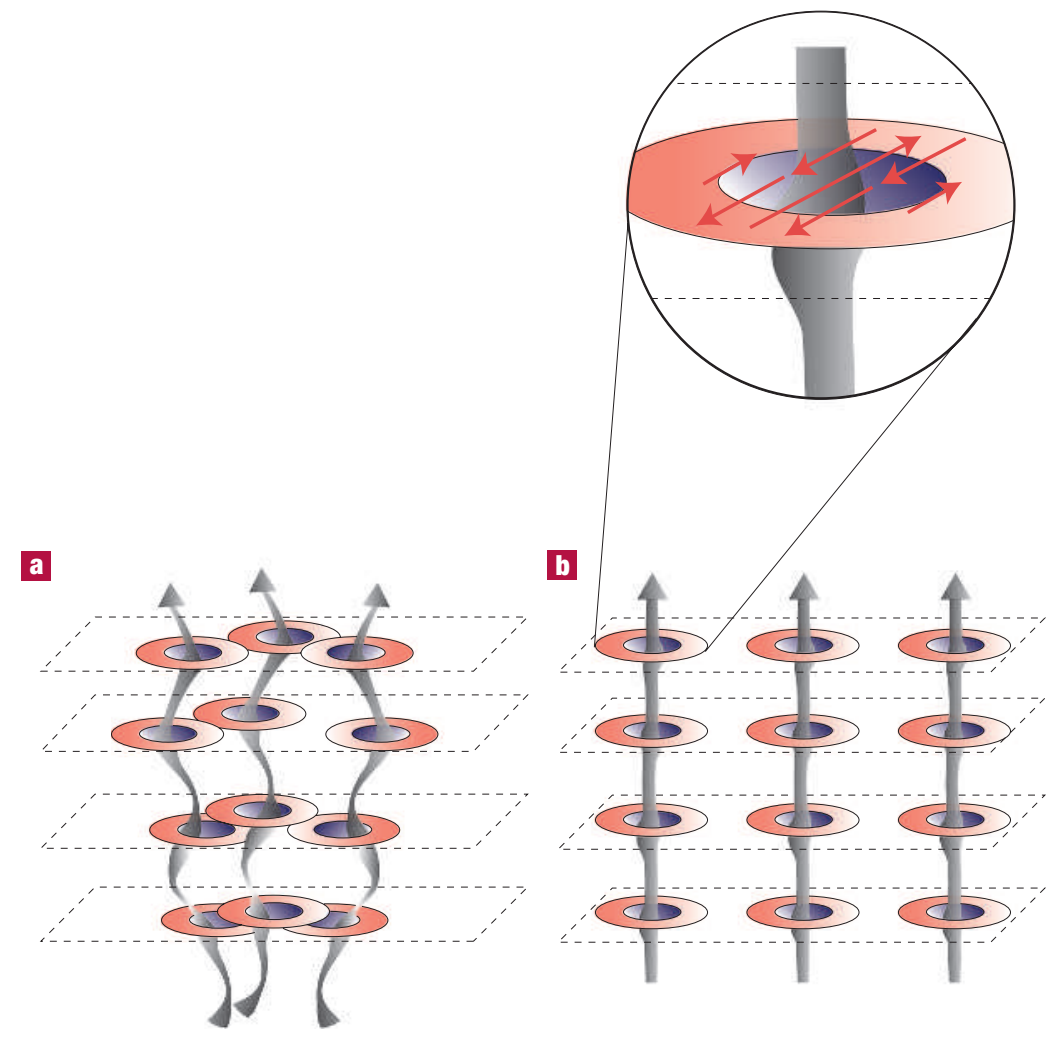

Figure 2 Flux lines in a hightemperature superconductor. a, A typical high-temperature semiconductor with a disordered lattice of flux lines with no alignment of the flux lines perpendicular to the planes. $\mathbf{b}$, The study of Lake et al. ${ }^{1}$ shows how a small applied magnetic field can lead to an ordered state. The flux lines are now straightened along the $c$-axis. Inset: The antiferromagnetic stripe phase near the flux lines is characterized by an 'incommensurate' spatial modulation of the antiferromagnetic spins. superconductivity is destroyed. To improve matters, the flux lines should be made sturdier by ordering the flux pancakes along the direction perpendicular to the planes (' $c$-axis'). Lake and colleagues propose that this is exactly what happens in the presence of a small external magnetic field.

Although the superconductivity is reasonably conventional, everything else in high-temperature superconductors is very unusual. The effects of interactions between electrons are extremely strong, and the conventional picture of the metallic state is of little, if any, use. Instead, one encounters phenomena of a highly collective nature. Various ordering tendencies, such as antiferromagnetism, seem to be in fierce competition with superconductivity, but this fight is ruled by the laws of quantum physics ${ }^{4,5}$. This is called 'quantum criticality', referring to the analogy with the phenomena found in the proximity of transitions between classical phases of matter.
When the competition is tight, a small advantage can make a big difference.

External magnetic fields are not good for superconductivity. They tend to cause a particular competing state to take over ${ }^{5}$. When the superconducting carriers come to a standstill and antiferromagnetism is restored, a 'stripe phase' is formed $^{6,7}$, in which antiferromagnetism occurs in an incommensurate form, characterized by spatial modulations of the antiferromagnetic spins (as shown in the inset of Fig. 2b). In particular, this should occur near the magnetic flux lines, where superconductivity is weak ${ }^{5}$.

With their neutron scattering experiments, Lake et al. show that this 'stripy' antiferromagnetism near the flux lines becomes surprisingly three dimensional if stabilized by an applied magnetic field. Stabilizing it by any other means would not lead to an alignment along the $c$-axis ${ }^{7}$. In a magnetic field, the flux lines apparently help the planar antiferromagnetic islands attached to the flux pancakes to align along the difficult $c$-axis. And to get these islands aligned along the $c$-axis the flux lines have to be straight (see Fig. 2b). Why is this good for technology? It works in both ways. When the flux lines mediate this three-dimensional spin order, these lines become in turn more stiff because it gives an energetically more favourable spin configuration. Therefore, the lattice moves less easily in a magnetic field and superconductivity is more stable.

Will trains start to levitate, at last? I am sceptical, because the 'stripy' forces aligning the flux lines are rather weak and, more importantly, serious quantum competitions of this kind are only found in superconductors with a relatively low critical temperature. The significance of this work lies more in the fundamental aspects. Much will be learned from the interplay of flux-dynamics and quantum criticality. In particular, the stripe phase is actually quite poorly understood, but thanks to Lake et al. it has now become possible to shake and push this enigmatic state by using the fancy tools of flux technology.

\section{REFERENCES}

1. Lake, B. et al. Nature Mater. 4, 658-662 (2005).

2. http://nobelprize.org/physics/laureates/2003

3. Blatter, G. et al. Rev. Mod. Phys. 66, 1125-1388 (1994)

4. Laughlin, R. B. A Different Universe Ch. 12 (Basic Books, New York, 2005)

5. Sachdev, S. \& Zhang, S. C. Science 295, 452-454 (2002).

6. Zaanen, J. et al. Phil. Mag. B 81, 1485-1531 (2001).

7. Kivelson, S. A. et al. Rev. Mod. Phys. 75, 1201-1241 (2003). 